\title{
Radioterapia esterotáxica: ¿Un segundo gold estándar en el tratamiento del cáncer de pulmón en etapa precoz?
}

\author{
ALEJANDRO SANTINI B.*, CRISTIÁN VALDEZ C.**, VERÓNICA SEPÚLVEDA A.*, \\ RICARDO BAEZA L.* y SERGIO BUSTOS C.**
}

\begin{abstract}
Stereotactic radiation therapy: a second gold standard in the treatment of early-stage lung cancer?
\end{abstract}

Lung cancer is still the leading cause of cancer death in the world. Although in Chile this is not the case, the northern regions of the country show higher incidence and mortality rates than the other Chilean regions. In recent years screening guides for lung cancer with low-dose scanner have begun to be established, and most of the medical societies involved in this subject have already settled the selection criteria. At the same time new techniques of treatment for these patients have developed, with highly sophisticated radiotherapy such as SBRT (Stereotactic Body Radiotherapy) and SBART (Stereotactic ablative body radiation therapy) that are revealing extremely encouraging results and augur significant changes in the coming years. In the present review we analyze the current work, their results, and the future of this treatment modality.

Key words: Lung Cancer; radiotherapy; stereotactic radiotherapy; ablative radiotherapy.

\section{Resumen}

El cáncer de pulmón continúa siendo la principal causa de muerte por cáncer en el mundo, si bien en Chile aun no lo es, en las regiones del norte del país ya es una tendencia, siendo su incidencia y mortalidad mayores a las del resto del país. En los últimos años se han publicado guías de screening de cáncer pulmonar con tomografía de baja intensidad y en ese sentido, la mayoría de las sociedades científicas vinculadas al tema, ya tienen establecido los criterios de selección para ello. En forma paralela en los últimos años se han desarrollado nuevas técnicas de tratamiento para estos pacientes, con radioterapia altamente sofisticada, como son la radioterapia esterotáxica (SBRT) y radioterapia ablativa (SBART) que están mostrando resultados muy alentadores y que auguran cambios muy significativos en los próximos años. En el presente artículo se revisan los trabajos actuales, los resultados y el futuro de esta modalidad de tratamiento.

Palabras clave: Cáncer de pulmón; radioterapia; radioterapia esterotáxica; radioterapia ablativa.

\section{Introducción}

El cáncer pulmonar es la principal causa de muerte por cáncer a nivel mundial. Según GLOBOCAN, el año 2012 fallecieron a nivel mundial, 1,8 millones de personas por esta enfermedad, lo que representa alrededor del 13\% de todos los cánceres (www.globocan.iarc.fr). En hombres, la mayor incidencia de esta enfermedad se centra en Europa Central y Oriente $(53,5$ casos por $100.000)$ y países orientales de Asia $(50,4$ por 100.000). Las menores incidencias se ubican en África. Sudamérica se ubica dentro de las regiones con menores incidencias de esta enfermedad, con una incidencia cercana a 20 por 100.000 habitantes/año. En mujeres, la incidencia es notoria-

Centro Oncológico Antofagasta, Chile:

* Oncólogo Radioterapeuta.

** Físico Médico. 
mente menor, pero con una distribución mundial ligeramente distinta, con mayor incidencia en Norteamérica y el norte de Europa ${ }^{1}$.

En Chile hay una gran variación a lo largo del país, en el norte es donde se da la mayor incidencia y mortalidad, y donde representa la principal causa de muerte por cáncer, siendo la incidencia de cáncer de pulmón en la región de Antofagasta, de las más altas de Sudamérica (41 por 100.000 en hombres, 18,6 en mujeres).

En la Figura 1 se realiza una comparación entre la Región de los Ríos y la Región de Antofagasta, con los datos de la última publicación de registro poblacional de cáncer Chile 2003-2007².

En cuanto al estadío en el momento del diagnóstico, hasta ahora esta neoplasia se diagnostica en etapas avanzadas. En Europa sólo el 20\% se diagnostican en etapas I y II, cuando es aún una enfermedad que teóricamente podría ser resecada, pero sólo la mitad de los pacientes en estas etapas están en condiciones de ser operados. El 80\% restante son pacientes con enfermedad avanzada; de ellos, el $40 \%$ tienen una enfermedad resecable de los cuales sólo el $20 \%$ son operables ${ }^{3}$. Por lo tanto, podemos decir que en los países desarrollados el $75 \%$ de los pacientes no son candidatos a la cirugía, ya sea por una enfermedad irresecable o por ser un paciente inoperable por comorbilidades.

Sin embargo, esta realidad parece estar cambiando a la luz de la puesta en marcha de planes de screening en cáncer de pulmón. Este screening, hecho con TAC de baja intensidad ha mostrado resultados bastante alentadores, y ya forma parte de las recomendaciones de la mayoría de las asociaciones oncológicas vinculadas al tema ${ }^{4-7}$. Las publicaciones disponibles han demostrado que este tamizaje, en pacientes de alto riesgo, reduce la tasa de mortalidad por cáncer de pulmón en un $20 \%$. Hasta el momento es la única herramienta que ha demostrado reducciones en mortalidad.

Estas campañas de screening seguramente cambiarán la epidemiología de la enfermedad y son un desafío para los sistemas sanitarios actuales, dado que llegarán un número cada vez mayor de pacientes asintomáticos, con lesiones pequeñas factibles de tratamientos curativos y con una buena tasa de control de la enfermedad.

Datos preliminares entregados por el Registro Hospitalario de Cáncer del Hospital Regional de Antofagasta, vigente desde el año 2011, han demostrado que en dicho hospital, el 93\% de los pacientes se diagnostican en etapas III y IV y sólo el $14 \%$ son tratados con intento curativo ( $5 \%$ cirugía y $9 \%$ radioterapia y quimioterapia concomitante). Estas cifras son verdaderamente alarmantes y esperamos que en los próximos años se vean mejoradas, aproximándonos a las cifras descritas en los países desarrollados.

Otro punto de importancia en las regiones del norte es la frecuencia de los diferentes tipos histológicos, siendo el carcinoma escamoso la principal forma anatomopatológica, representando más de las dos terceras partes de los casos. (Registro Hospitalario de Cáncer Hospital Regional de Antofagasta).

El tratamiento de elección, para aquellos pacientes con cáncer de pulmón en etapas tempranas es la cirugía (lobectomía/neumonectomía + disección ganglionar hilio-mediastinal) y en los pacientes con mayor riesgo se plantean resecciones más conservadoras como la resección en cuña ${ }^{8}$. La radioterapia curativa hasta hace dos o tres años estaba relegada a aquellos pacientes que no eran candidatos a una cirugía.

Según la revisión de Krost, del Reino Unido, la sobrevida a 5 años en pacientes operados es, para el estadío I, 76\% (T1 N0 M0 $=84 \%$, T2 N0 M0 $=68 \%$ ), para estadío II, $47 \%$ (T1-T2 N1 $\mathrm{M} 0=47 \%$, T3 N0 M0 con invasión de pared toráxica $56 \%$, invasión mediastinal $29 \%$, invasión de bronquio proximal $36 \%)^{9}$.

En aquellos pacientes que no son candidatos a cirugía y que no reciben ningún tratamiento la sobrevida media es de 13 meses para los T1 y de 8 meses para los T2, y la sobrevida a 5 años es del $16 \%{ }^{10}$.

La radioterapia convencional implica un tratamiento que entrega una dosis de 60-70 Gy en 30-35 fracciones durante 6 a 7 semanas. A pesar de utilizar tratamientos sofisticados, como los tratamientos en tres dimensiones o IMRT (radioterapia de intensidad modulada), los resultados han sido francamente inferiores a los de la cirugía, con una tasa de recurrencia local de hasta el $70 \%{ }^{11-12}$.

En un intento por mejorar estos resultados, hace 20 años se planteó la estrategia de combinar un tratamiento esterotáxico, con tratamientos hipofraccionados de dosis altas. En general entre 1 y 5 fracciones entregadas en un período menor a dos semanas.

Esta técnica se empleó inicialmente en la década de 1960 para lesiones intracerebrales y dado que fue desarrollada por neurocirujanos, se la llamó radiocirugía. Su empleo en cáncer broncopulmonar comienza en 1995, con resultados que han sido cada vez más alentadores ${ }^{13}$.

En los últimos años se han publicado varios trabajos donde se emplea Radioterapia Esterotáxica Corporal, (denominada en EE. UU. Stereo- 
Incidenciay Mortalidad (2003-2009) por cáncer en Hombres

según localización. Chile RPC Región de Los Ríos 2003-2007.

Fuente: Elaboradopor Unidad VENTy EStudios. Depto. Epidemiobgia, DPRLAS-MINSAL y RPC de Los Rios.

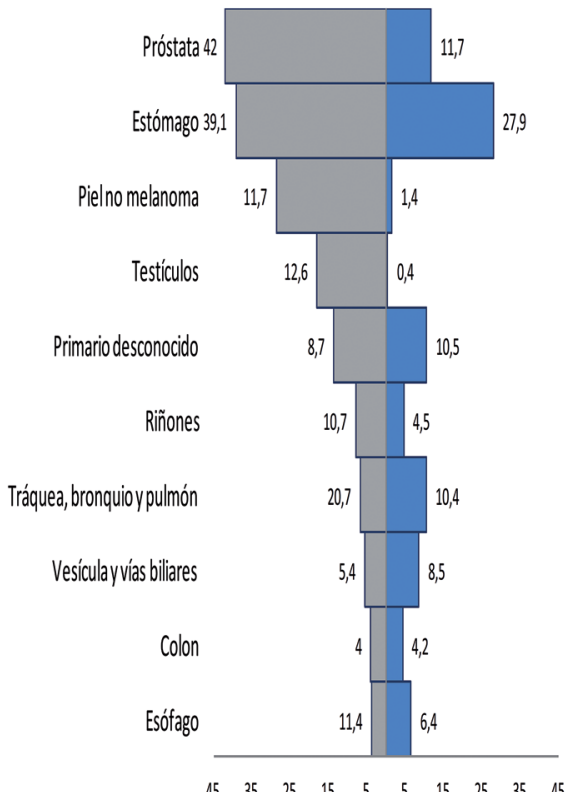

口incidencia $\square$ Mortalidad

Incidencia y Mortalidad (2003-2007) por cáncer en Hombres según localización.

Chile RPC Región de Antofagasta 2003-2007 (Tasa por 100.000 Hbts).

Fuente: Elaborado por Unidad VENTy Estudios. Depto. Epidemiologia, DIPLASMINSAL yRPC de Antofagasta

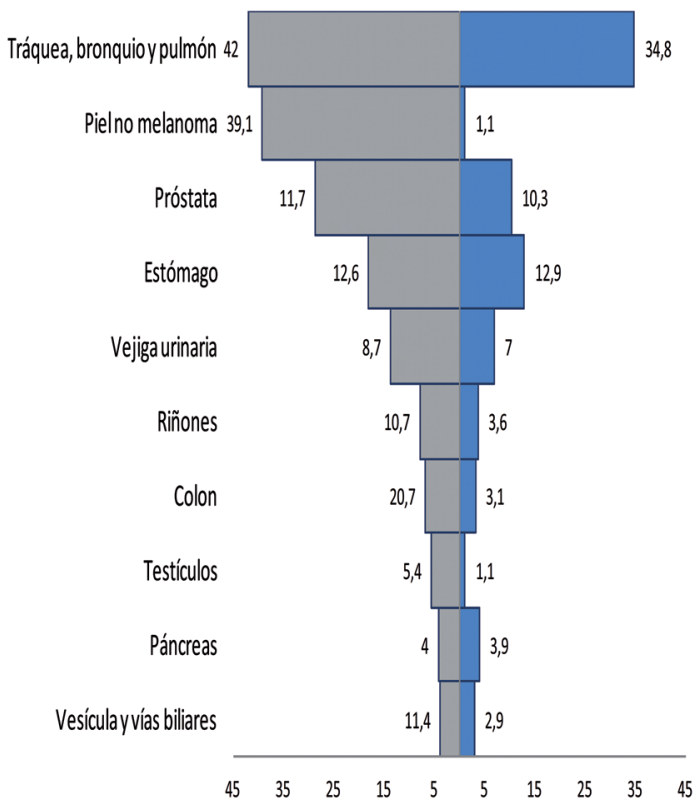

Incidenciay Mortalidad (2003-2007) porcáncer en Mujeres

según localización. Chile RPC Región de Los Ríos 2003-2007.

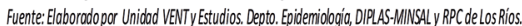

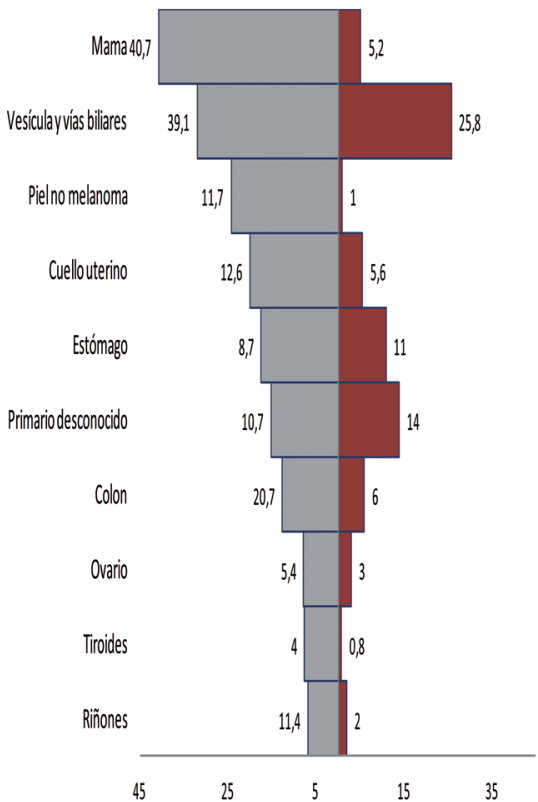

TIncidencia Mortalidad

Incidencia y Mortalidad (2003-2007) por cáncer en Mujeres según localización.

Chile RPC Región de Antofagasta 2003-2007 (Tasa por 100.000 Hbts).

Fuente: Elaborado por Unidad VENTy Estudios. Depto. Epidemiologia, DIPLAS-MINSAL y RPC de Antofagasta

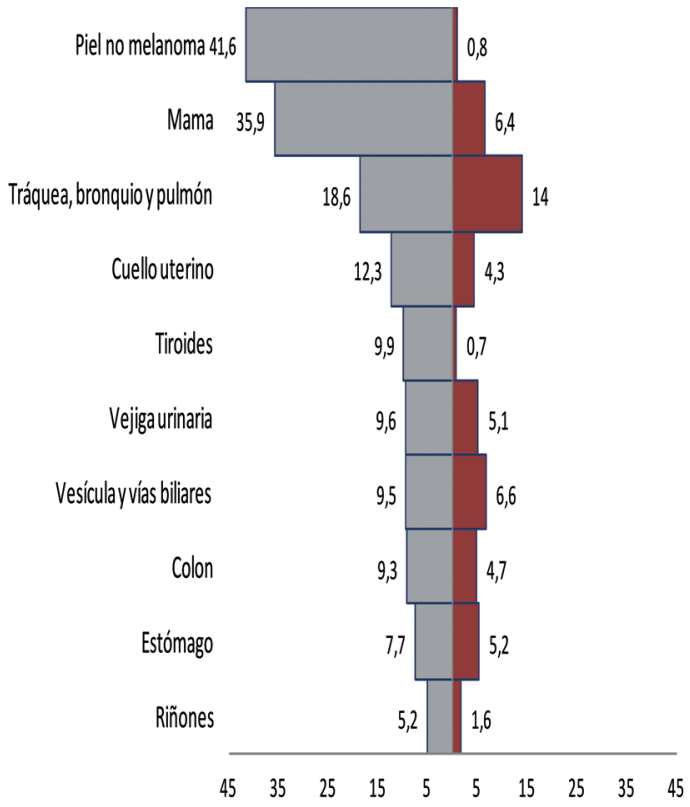

Figura 1. Comparación de incidencia y mortalidad por cáncer entre la Región de los Ríos y la Región de Antofagasta, para hombres y mujeres. Nótese que en Antofagasta, en hombres, es la primera causa de mortalidad y en Los Ríos la $4^{\circ}$ y no figura entre las 10 primeras en mujeres. 
tactic Body Radiotherapy: SBRT) o Radioterapia Esterotáxica Ablativa (denominada en Europa Stereotactic ablative body radiation therapy: $S A$ $B R T$ ) con resultados extremadamente alentadores. Actualmente no sólo es la técnica de radioterapia más adecuada para tratamiento de pacientes con cáncer de pulmón no células pequeñas y no candidatos a cirugía, sino que ya se ha posicionado, para algunos autores, como un segundo "gold standard"14. SBRT y SABRT son utilizados como sinónimos en esta publicación.

En una reciente publicación, Valle y cols. revisan las conductas de tratamiento en los centros que participan en la NCCN (National Comprehesive Cancer Network) que se emplearon en los pacientes con cáncer de pulmón no células pequeñas en etapa I tratados entre 2007 y 2011 . En ese período el $12 \%$ de los pacientes se trataron con SBRT, con una gran variación de las conductas entre los diferentes centros, lo que refleja una falta de evidencia nivel I al respecto a las recomendaciones ${ }^{64}$.

Como mencionamos anteriormente, en los últimos años se han publicado resultados que demuestran un rol muy importante del TAC de baja intensidad en el diagnóstico oportuno y en el screening de cáncer de pulmón ${ }^{15}$. En varias guías clínicas ya existe una recomendación clara para este estudio, por lo que cabe esperar que en un futuro, también en Chile sea una práctica habitual $\mathrm{y}$ comencemos a recibir pacientes con cáncer de pulmón en etapas más tempranas con mucha mayor frecuencia. Esto plantea un desafío para los centros públicos y privados de radioterapia existentes en el país, ya que el contar con una alternativa de tratamiento que se perfila con un menor costo, que se hace en forma ambulatoria y con una efectividad similar, demandaría un esfuerzo para implementarla.

Senan y cols., de la UV University de Amsterdam, demostraron recientemente, que la SBRT es una alternativa válida para el tratamiento de pacientes con cáncer pulmonar diagnosticado en el marco de los nuevos estudios de screening con TAC de baja intensidad ${ }^{17}$.

\section{Aspectos radiobiológicos de la SBRT}

La característica fundamental de la SBRT es el empleo de dosis muy altas de radiación en muy pocas fracciones, lo que implica entregar una dosis equivalente biológica (BED) de más de 100 Gy. La BED es un valor de dosis, empleado en radiobiología y que no sólo tiene en cuenta la dosis total entregada, sino que además la dosis que se aplica en cada fracción, en número de fracciones y el tiempo total del tratamiento. En ese sentido no es lo mismo 20 Gy en una fracción que 20 Gy en 10 fracciones, el efecto biológico del primer esquema es mucho mayor que el del segundo. Es una herramienta que nos permite comparar los efectos de diferentes fraccionamientos en distintos tejidos. En los estudios retrospectivos de SBRT en cáncer localizado de pulmón no células pequeñas, se ha demostrado que los resultados más positivos se dan cuando la BED de los tratamientos es mayor de $100 \mathrm{~Gy}^{18,19}$.

En los últimos años se ha investigado profundamente la radiobiología de este tipo de tratamientos ${ }^{18}$.

Desde hace muchos años sabemos que la muerte celular que sucede luego de un tratamiento estándar de radiación, es consecuencia del daño a la doble cadena de ADN, que lleva a una muerte reproductiva, es decir las células mueren después de algunas divisiones celulares luego de aplicada la radiación. Sin embargo, este mecanismo, por sí solo no explica los resultados obtenidos con $\mathrm{SBRT}^{20}$. Hoy sabemos que se ponen en marcha otros mecanismos diferentes a las clásicas 4 "Rs" de la radiobiología: Reparación, Reclutamiento, Reoxigenación y Repoblación.

Si bien aún no se ha logrado demostrar fehacientemente los mecanismos íntimos que acontecen con el empleo de dosis altas e hipofraccionadas de radiación, la mayoría de los autores afirman que la muerte celular se debe a dos mecanismos fundamentales. Primero, una muerte indirecta debido a daño vascular masivo, que secundariamente produce el daño celular por hipoxia. Y en segundo lugar, a la puesta en marcha de una cascada inmunológica donde están implicados un sinnúmero de moléculas inmunomoduladoras, tales como el complejo mayor de histocompatibilidad, moléculas de adhesión, factores quimiotácticos y otros como el factor de necrosis tumoral o interleuquinas ${ }^{20,21}$.

Se ha demostrado claramente en modelos tumorales y en tumores in vivo, que luego de una fracción de 10 Gy hay una casi desaparición de todo el árbol vascular a las pocas horas de la aplicación ${ }^{20}$. Esto implica indefectiblemente muerte masiva de células tumorales.

El segundo efecto inmunomodulador de la radioterapia con dosis altas, tendría a su vez dos componentes diferentes. El primero es el efecto de la radiación dentro de la zona tratada o blanco de tratamiento, que está vinculado a una sensibilización directa de las células tumorales a la muerte celular mediada por células $T^{21}$. Se han documentado varios mecanismos a través de los 
cuales la SBRT puede incrementar la sensibilidad de las células tumorales mediada por células $\mathrm{T}$, entre ellos: una superregulación de todos los elementos vinculados, la expresión de las proteínas de membrana y del complejo mayor de histocompatiilidad, un incremento de la expresión de antígenos tumorales, la superregulación de ligandos que coactivan a las células $\mathrm{T}$, etc ${ }^{21}$. El segundo efecto es por fuera de la zona irradiada $y$ es conocido desde hace años como el efecto "abscopal" de la radioterapia.

En conjunto podemos concluir que fracciones altas de radiación causan una muerte celular directa e indirecta, que conduce un masivo lanzamiento de antígenos tumorales lo que desencadena una respuesta inmunológica masiva contra el tumor. Este mismo efecto explicaría el ya conocido efecto abscopal, demostrado hace ya varias décadas ${ }^{37}$. Sin duda que los avances en el empleo de toda una nueva batería de drogas inmunomoduladoras en conjunto con esta forma de tratamiento aparecen como una estrategia sumamente alentadora ${ }^{23,24,65}$.

\section{Aspectos técnicos de la SBRT}

El empleo de SBRT requiere de un alto nivel de control de calidad en todo el proceso de tratamiento. Esta seguridad debe aplicarse desde la indicación hasta la ejecución del tratamiento, para lo cual es fundamental la integración de la imagenología moderna, la simulación, la planificación dosimétrica, y la entrega de la dosis en el blanco prescrito ${ }^{25}$. Es de suma importancia contar con todo un equipo profesional comprometido y adiestrado para que los resultados sean los óptimos.

El proceso comienza con la inmovilización del paciente durante la simulación, que permitirá se produzca un mínimo movimiento durante el tratamiento (intrafracción), una mayor reproducibilidad (interfracciones) y una máxima confortabilidad durante el mismo ${ }^{26-66}$ (Figura 2).

El paso siguiente es la adquisición de las imágenes de TAC, teniendo especialmente en cuenta, el movimiento fisiológico del tumor durante la respiración (imágenes en 4D). De esta manera podemos adecuar los márgenes del mismo según su movimiento (es diferente el margen cráneocaudal que el lateral o el dorso-ventral). Este punto es de crucial importancia dado que los márgenes deben variar de acuerdo al movimiento del tumor. Idealmente se debe emplear un TAC en cuatro dimensiones (4DCT) aunque se puede utilizar CT (tomografía computarizadas) seriadas, en diferentes fases de la respiración. En aquellos casos donde la amplitud de movimiento es importante, el incluir toda la zona de excursión tumoral puede dar a lugar a complicaciones tardías importantes, por lo que se debe emplear alguna técnica de manejo de la respiración. Estas técnicas incluyen implementos de compresión abdominal, equipos de sincronización que hacen que el acelerador lineal "dispare" durante una determinada etapa de la respiración (gatting), asumir el movimiento normal durante la respiración y agregar un mayor margen para compensarlo (ITV), intentar que el

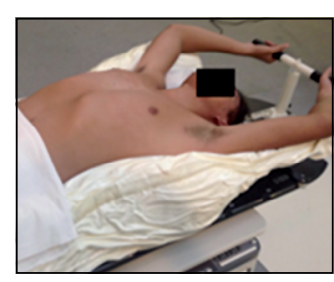

Inmovilización

- Reduce la probabilidad de errores de posicionamiento

- Ayuda a la comodidad del paciente, mejorando la cooperación de éste en el equipo de tratamiento

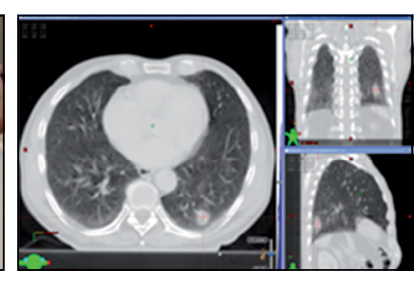

Adquisición de imágenes

- Utilizado para realizar modelo 3D del paciente

- Identificar movimiento tumoral debido a la respiración

- Decisión de manejo respiratorio en caso de ser necesario

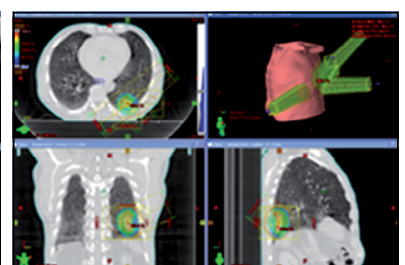

\section{Planificación}

- Definición de órganos sanos

- Simulación de las condiciones de tratamiento en el paciente

- Información utilizada para decidir la mejor estrategia de tratamiento

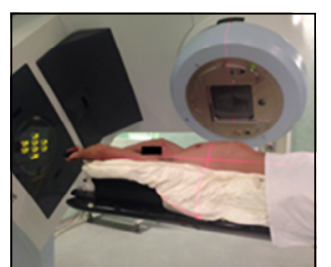

Tratamiento

- Verificación del posicionamiento mediante imágenes en cada tratamiento

- Manejo de la respiración en caso de ser necesario

Figura 2. Proceso de inmovilización, simulación o adquisición de imágenes, planificación y tratamiento de SBRT (las imágenes son cortesía del Centro Oncológico Antofagasta). 

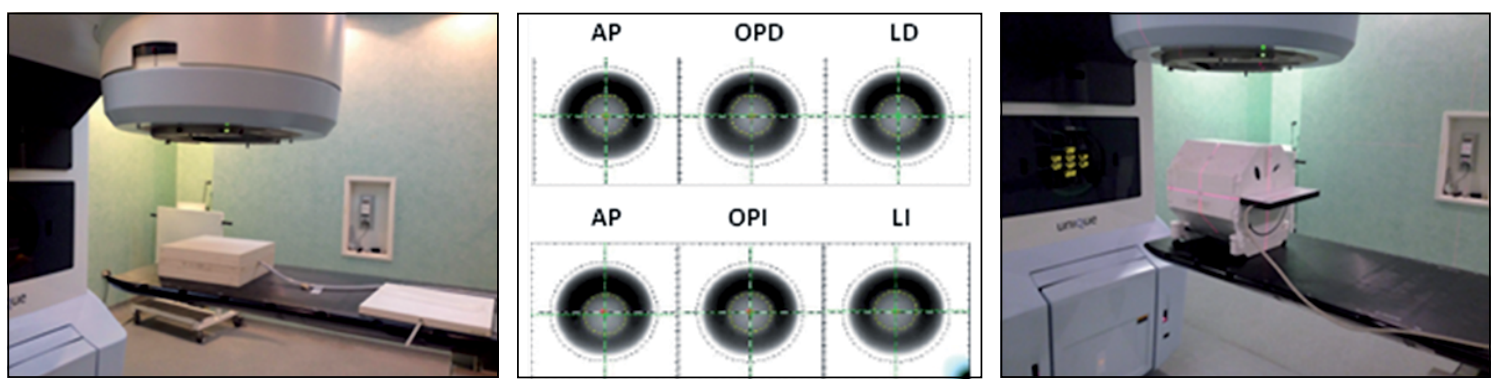

Figura 3. Control de calidad en tratamientos SBRT; izquierda, control de dosis absoluta con cámara de ionización; centro, control de tamaño de isocentro; derecha, análisis de distribución de dosis 2D (las imágenes son cortesía del Centro Oncológico Antofagasta).

paciente mantenga la respiración durante un determinado tiempo o mantener un movimiento del haz de radiación, acompañando la respiración en tiempo real (tracking) $)^{66}$.

Toda esta técnica requiere un control de calidad estricto que garantice la entrega óptima del tratamiento prescrito (Figura 3).

Para la entrega de dosis, se emplean diferentes técnicas, generalmente con múltiples campos de tratamiento, o con técnicas dinámicas como VMAT (Volumetric Modulated Arc Therapy) o arcoterapia. SBRT puede emplearse con aceleradores lineales con herramientas que permiten monitorear el movimiento de las lesiones, o con equipos especialmente diseñados a tal fin como CiberKnife, ambos equipos obtienen resultados similares.

\section{Revisión de la literatura, estudios retrospectivos}

Hasta hace algunos meses la indicación principal de SBRT en cáncer de pulmón no células pequeñas, era para los pacientes con lesiones tempranas (T1-T2), sin linfonodos positivos pero que tenían una contraindicación para la cirugía o que se negaban a un tratamiento quirúrgico por el riesgo de morbimortalidad que este implica ${ }^{27}$. Sin embargo, durante los últimos dos años se han publicado varios estudios con resultados muy alentadores por lo que para varios autores en la actualidad se podría hablar de un doble "Gold standard" 14 .

Senan y cols. analizan los resultados de los tratamientos de cirugía y SBRT en pacientes con cáncer de pulmón en etapas tempranas y detectados con TAC de baja intensidad. Estos autores plantean ventajas y desventajas de cada uno de los tratamientos ${ }^{17}$ (Tabla 1).

Varios estudios retrospectivos muestran tasas de control local con SBRT mayores al $80 \%$ con una muy baja toxicidad en pacientes con $\mathrm{T} 1$ y T2 periféricos (Tabla 2).

Recientemente Yu y cols. publican un exhaustivo análisis retrospectivo sobre la efectividad de la SBRT o la cirugía en pacientes con cáncer de pulmón temprano ${ }^{28}$. Estos autores del departamento de Radioterapia de la Universidad de Yale, analizan la base del SEER (The Surveillance, Epidemiology, and End Results Program of the National Cancer Institute) e incluyen pacientes mayores de 67 años tratados entre 2007 y 2009.

Tabla 1. Ventajas y desventajas de SBRT y cirugía en el tratamiento de pacientes con cáncer de pulmón en etapas tempranas diagnosticados con estudios de screening

\section{Cirugía}

- Ventajas

- Diagnóstico patológico definitivo

- Permite un diagnóstico de compromiso linfoganglionar

- Permite adecuar un tratamiento adyuvante en caso de linfonodos $(+)$

- Desventajas

- Procedimiento con morbi-mortalidad elevada

- Procedimiento invasivo en pacientes en los que posiblemente no tengan una enfermedad maligna y que muchas veces tienen patologías asociadas

\section{SBRT}

- Ventajas

- Sobrevida a 5 años mayor al 90\%

- Tratamiento ambulatorio

- Preservación de función pulmonar y calidad de vida

- Mínima morbilidad y casi cero mortalidad

- Desventajas

- No hay una verificación patológica definitiva

- La fibrosis local por radioterapia puede enmascarar una recurrencia local

Modificado de Senan y cols ${ }^{17}$. 
Tabla 2. Estudios retrospectivos de SBRT en pacientes con cáncer de pulmón no células pequeñas (CPNCP) en etapas precoces

\begin{tabular}{|c|c|c|c|c|}
\hline Estudio & $\stackrel{n}{\text { pacientes }}$ & $\begin{array}{l}\text { Esquema de } \\
\text { tratamiento }\end{array}$ & Control local & Complicaciones \\
\hline Van der Voort ${ }^{32}$ & 70 & 3 x 12-15 Gy & $96 \%$ a 2 años & Toxicidad tardía en el $10 \%$ \\
\hline Brown y col' ${ }^{33}$ & 59 & $\begin{array}{l}1-5 \text { fracciones } \\
(15-67 \mathrm{~Gy})\end{array}$ & $\begin{array}{l}\text { Sobrevida libre de } \\
\text { enfermedad } 90 \%\end{array}$ & Neumonitis G3 en $7 \%$ \\
\hline Senthi y cols ${ }^{34}$ & 676 & $\begin{array}{l}3-8 \text { fracciones } \\
(54-60 \mathrm{~Gy})\end{array}$ & $89 \%$ a 5 años & \\
\hline Onishi y cols ${ }^{35}$ & 257 & $\begin{array}{l}\text { 1-14 fracciones } \\
(30-84 \text { Gy) }\end{array}$ & $\begin{array}{l}84 \% \text { a } 5 \text { años } \\
(\mathrm{BED}>100 \mathrm{~Gy})\end{array}$ & $\begin{array}{l}\text { Complicaciones pulmonares G3 5,4\% } \\
\text { Complicaciones esofágicas } 1 \%\end{array}$ \\
\hline Nagata y cols ${ }^{36}$ & 104 & 4 x 12 Gy & $\begin{array}{l}\text { Sobrevida libre de } \\
\text { progresión a } 3 \text { años } 69 \%\end{array}$ & $\begin{array}{l}\text { Disnea grado III } 9 \% \\
\text { Neumonitis } 7 \% \\
\text { Dolor } 2 \%\end{array}$ \\
\hline
\end{tabular}

Se revisaron 367 pacientes tratados con SBRT y 711 con cirugía. La toxicidad aguda (de 0 a 1 mes) fue de $7,9 \%$ para SBRT versus $54,9 \%$ para cirugía $(\mathrm{p}<0,001)$. A los 24 meses del tratamiento la diferencia en favor de la SBRT no era significativa $(69 \%$ versus $73,9 \% \mathrm{p}=0,31)$. El IRR de la toxicidad para SBRT versus Cirugía fue 0,74 (95\% CI de 0,64-0,87). La mortalidad global fue menor para la SBRT $(40,1 \%$ versus $22,3 \%$, $\mathrm{p}<0,001)$. Las complicaciones descritas para la cirugía fueron: infarto agudo del miocardio, arritmias cardíacas, trombosis venosa profunda, tromboembolismo pulmonar, etc. Todos los pacientes sometidos a cirugía requieren una hospitalización, de al menos 3 días si no hay complicaciones, mientras que la SBRT es ambulatoria y consiste en 1 a 5 fracciones de radioterapia que implican entre 1 y $2 \mathrm{~h}$ cada una.

Inicialmente los autores seleccionaban pacientes con lesiones periféricas debido a que el análisis de los efectos adversos parecía diferente entre estos y las lesiones más centrales ${ }^{29-31}$, incluso se había planteado una zona de exclusión ("no fly zone") para tratamiento como se observa en la Figura 4. Sin embargo, actualmente las restricciones son cada vez menores cuando se adecua el fraccionamiento.

En los primeros estudios, en lesiones más centrales se describieron algunos casos de toxicidad severa a largo plazo ${ }^{38-39}$. Sin embargo, en estudios más recientes y adecuando el fraccionamiento, aumentado el número de fracciones los resultados son similares a los que se observaban en los pacientes con tumores periféricos. En la Tabla 3 de describen algunos de los estudios más modernos donde se incluyen pacientes con tumores centrales.

Recientemente, Baba y cols. realizaron una

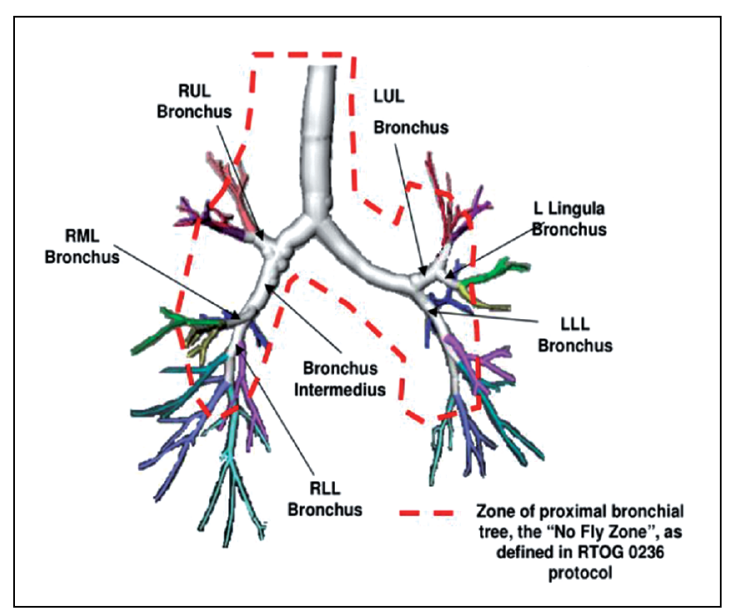

Figura 4. Zona de exclusión, para SBRT recomendada originalmente por el RTOG (Radiation Therapy Oncology Group). Sin embargo, en la actualidad se hacen tratamientos en dicha zona con ligeros cambios en el fraccionamiento $^{46}$.

revisión de 20 estudios con más de 500 pacientes con lesiones centrales tratadas con SBRT. La toxicidad grado 3-4 fue del 8,6\% y la mortalidad vinculada al tratamiento fue del $2,7 \%$, que si bien es relativamente baja es algo más alta que la que se reporta en lesiones más periféricas. El control local a tres años fue del $60-100 \%$ y la sobrevida total del $50-75 \%{ }^{46}$.

En las recomendaciones de NCCN (National Comprehensive Cancer Network) de 2015, se plantean diferentes esquemas de fraccionamiento, según localización siendo los tumores centrales, a menos de $2 \mathrm{~cm}$ de los bronquios principales tratados en forma más conservadora con dosis fracción algo menor y con mayor número de fracciones $^{7}$ (Tabla 4). 
Tabla 3. Resultados de SBRT en pacientes con cáncer de pulmón no células pequeñas (CPNCP) con lesiones centrales

\begin{tabular}{|c|c|c|c|c|c|}
\hline Autor & $\begin{array}{l}\text { Pacientes } \\
\text { (n) }\end{array}$ & $\begin{array}{c}\text { Características del } \\
\text { tumor }\end{array}$ & Dosis fracción & Control local & Sobrevida \\
\hline Chang y col' ${ }^{40}$ & 27 & $\begin{array}{l}\mathrm{T} 1-\mathrm{T} 248 \% \\
\text { Recurrencias 52\% }\end{array}$ & $40-50 \mathrm{~Gy} / 5 \mathrm{Fr}$ & $\begin{array}{l}3 \text { pacientes } \\
(40 \mathrm{~Gy})\end{array}$ & - \\
\hline Milano y col ${ }^{41}$ & 53 & $\begin{array}{l}\text { T1-T2 } 66 \% \\
\text { CPNCP metastásico 36\% }\end{array}$ & $20-55 / 1-18 \mathrm{Fr}$ & $73 \%$ a 3 años & $\begin{array}{l}72 \% \text { a } 2 \text { años } \\
(\mathrm{T} 1-\mathrm{T} 2)\end{array}$ \\
\hline Haasbeek y col ${ }^{42}$ & 63 & T1-T3 N0 & $60 \mathrm{~Gy} / 8 \mathrm{Fr}$ & $92,6 \%$ a 5 años & $\begin{array}{l}\text { SLE } 71 \% \\
\text { SVT } 49,7 \%\end{array}$ \\
\hline Rowe y col ${ }^{43}$ & 47 & $\begin{array}{l}\text { T1-T2 59\% } \\
\text { CPNCP } 41 \%\end{array}$ & $50 \mathrm{~Gy} / 4 \mathrm{Fr}$ & 2 fallos local & SLP $24 \%$ a 2 años \\
\hline Oshiro y col ${ }^{44}$ & 21 & $\begin{array}{l}\text { Recurrencias de CPNCP } \\
95 \%\end{array}$ & $25-39 \mathrm{~Gy} / 1-10 \mathrm{Fr}$ & $60 \% 2$ años & SVT $62,2 \%$ a 2 años \\
\hline Unger y col ${ }^{45}$ & 20 & CPNCP metastásico 85\% & $30-40 \mathrm{~Gy} / 5 \mathrm{Fr}$ & $63 \%$ a 1 año & SVT $54 \%$ a 2 años \\
\hline
\end{tabular}

$\mathrm{SLE}=$ Sobrevida libre de enfermedad ; SVT= Sobrevida total; SLP = Sobrevida libre de progresión.

Tabla 4. Fraccionamientos en SBRT (modificado de NCCN) ${ }^{7}$

\begin{tabular}{|c|c|c|}
\hline Dosis (Gy) & n fracciones & Indicación \\
\hline $25-34$ & 1 & Tumores periféricos, pequeños $(<2 \mathrm{~cm})$, y a más de $2 \mathrm{~cm}$ de la pared toráxica \\
\hline $45-60$ & 3 & Tumores periféricos, pequeños a menos de $2 \mathrm{~cm}$ de la pared torácica \\
\hline $48-50$ & 4 & $\begin{array}{l}\text { Tumores centrales o periféricos, menores de } 4-5 \mathrm{~cm} \text { y a menos de } 1 \mathrm{~cm} \text { de la pared } \\
\text { torácica }\end{array}$ \\
\hline $50-55$ & 5 & Tumores centrales o periféricos a menos de $1 \mathrm{~cm}$ de la pared torácica \\
\hline $60-70$ & $8-10$ & Tumores centrales \\
\hline
\end{tabular}

\section{Cirugía o SBRT}

Hasta la fecha no se han publicado trabajos randomizados que comparen cirugía con SBRT en pacientes operables, por lo que las recomendaciones que se pueden tomar son en base a estudios retrospectivos y series de casos.

Recientemente se realizó una revisión de 19 publicaciones, que analiza los resultados obtenidos en pacientes con riesgo alto para la cirugía, donde el procedimiento quirúrgico fue más conservador (resección sublobar) o SBRT ${ }^{47}$. Esta revisión nos muestra un control local del 90\% con SBRT, resultados similares a los obtenidos con una lobectomía en pacientes con bajo riesgo, pero mucho mejores que con la cirugía sublobar (resección en cuña). La tasa de recurrencia local fue del $4 \%$ en SBRT versus $20 \%$ de la cirugía $(\mathrm{p}=0,07)$.

Respecto a los resultados de la SBRT en pacientes de bajo riesgo quirúrgico, los datos disponibles son de la comparación entre estudios de pacientes que rechazaron la cirugía y eligieron SBRT. Hasta el momento hemos encontrado sólo tres estudios, que en conjunto suman más de 260 pacientes. En ellos, la tasa de control de la enfermedad local fue del 93\% para los T1 y de 73\% para T2. La sobrevida a 5 años fue de $72 \%$ y $62 \%$ respectivamente y la recurrencia local y a distancia fue del $20 \%{ }^{19,48,49}$. Estas cifras son similares a las publicadas en estudios de cirugía estándar en cáncer de pulmón.

En un reciente meta-análisis publicado por Zheng y cols., en el año 2014, se revisan los trabajos publicados entre el 2000 y $2012^{50}$. Se incluyeron 40 trabajos de SBRT, 30 de ellos retrospectivos (4.800 pacientes) y 23 estudios de cirugía, todos retrospectivos (7 a 50 pacientes). La edad media de los pacientes fue de 74 años y 66 años (SRBT y Cirugía respectivamente). La sobrevida a 1 año fue $83,4 \%$ versus $92,5 \%$, a 2 años $56,6 \%$ versus $77 \%$, y a 5 años 41,25 versus $66,1 \%$. Estos resultados inicialmente parecieran mostrar una leve ventaja para la cirugía; sin 
embargo, cuando se ajustan sólo los pacientes operables y por edad, la sobrevida libre de enfermedad y sobrevida total para ambos tratamientos es totalmente similar. Esto nos demuestra claramente que hay una selección de pacientes, que los más jóvenes y con menos comorbilidades son tratados con cirugía y los de mayor edad y más enfermos con SRBT, pero cuando las características de los pacientes son similares esas diferencias se pierden.

Para intentar comparar directamente estas dos técnicas, se plantearon tres estudios prospectivos randomizados, el "STAR", un trabajo cooperativo internacional, dirigidos por el equipo del MD Anderson, el "ROSEL", un grupo alemán y holandés, y otro del RTOG ${ }^{51,52,53}$. En ellos se incluían pacientes con cáncer de pulmón no células pequeñas etapa I, se comparan la lobectomía con disección hilio-mediastinal versus SBRT.

Los tres estudios fueron cerrados antes de tiempo por la dificultad en la inclusión de pacientes a la randomización. Esta situación se dio durante la firma del consentimiento, los pacientes raramente optaban por ser randomizados para evaluar dos tratamientos que de antemano no eran uno mejor que el otro, pero en uno se requería una toracotomía y en el otro no; la mayoría de los pacientes elegían la opción no quirúrgica.

Chang y cols., hicieron una revisión de los pocos pacientes incluidos en dos de estos ensayos y analizaron los resultados ${ }^{54}$.

Se incluyeron sólo 58 pacientes (31 SBRT y 27 cirugía), el seguimiento medio fue de 40,2 meses y la sobrevida estimada a 3 años fue de 95\% para SBRT, versus $79 \%$ para cirugía (HR $0,14$ y $\mathrm{p}=0,037)$, la sobrevida libre de enfermedad fue de $86 \%$ en SBRT versus $80 \%$ en cirugía $(\mathrm{p}=0,54)$.

En el grupo de SBRT el 10\% (tres pacientes) presentaron eventos adversos (dolor toráxico $10 \%$, disnea o tos $6 \%$, uno fatiga y otro fractura costal). En el grupo quirúrgico se describe un caso de muerte por complicaciones (4\%), 44\% de complicaciones G 3-4 (12 pacientes). Estos autores concluyen que, si bien el número de pacientes es pequeño y se requiere de un número mayor de pacientes, la SBRT es una opción válida de tratamiento para este grupo de pacientes.

En este sentido, Nieder y cols., se preguntan si este fallo en la inclusión de pacientes mantiene la interrogante planteada o es ya, una respuesta en si misma ${ }^{55}$.

Algunos autores, van más allá en este tema, como Rusthoven y cols., quienes plantean que estos resultados nos muestran que en cáncer de pulmón no células pequeñas se avecinan cambios similares a lo que sucedió al inicio de los tratamientos conservadores en el cáncer de mama en la década del ' 80 , o lo que sucede con el cáncer de próstata localizado y de bajo riesgo donde la radioterapia y la cirugía son alternativas válidas sin diferencias significativas entre ambas, a pesar de que no hay tratamientos randomizados que las comparen $^{56,67}$

En la actualidad hay dos estudios en curso, el RTOG-013 ${ }^{57}$, que analiza un escalamiento de dosis en tumores centrales menores de $5 \mathrm{~cm}$, y el RTOG-0915 que compara diferentes esquemas de tratamiento, 34Gy/1 fracción versus $48 \mathrm{~Gy}$ en 4 fracciones ${ }^{58}$. Se encuentran en preparación, y probablemente se comenzaron a enrolar pacientes a finales de 2015; ellos son VALOR (Veterans Affair Lung Cancer Surgery Or Sterotactic Radiotherapy) en USA y SBARTooth, en UK que intentarán responder varias interrogantes (citado por Chan y cols ${ }^{54}$ ).

Un último punto a analizar es la necesidad o no de contar con una confirmación histológica, previo al tratamiento de SBRT. Para los pacientes que son sometidos a cirugía por ser portadores de una lesión solitaria, la biopsia previa no se hace necesaria porque la probabilidad de malignidad es mayor del $65 \%$ y el riesgo de complicaciones de una biopsia por punción es elevada ${ }^{59}$. Esta situación es de mayor importancia en los pacientes que son sometidos a SBRT que generalmente tiene mayor tasa de comorbilidades ${ }^{60,61}$. Es por ello que publicaciones recientes analizan la necesidad o no de contar con una biopsia previa a la SBRT. Se han elaborado algoritmos, que utilizan uno o dos TAC seriados que analizan la evolución de la imagen, sumado al empleo del PET CT (TAC de emisión de positrones) con lo que se logra un factor predictivo positivo muy importante, y todo esto apoyado en el hecho de que el análisis de sobrevida de pacientes tratados con y sin biopsia muestra resultados similares ${ }^{62}$.

\section{Conclusión}

En el estudio y tratamiento del cáncer de pulmón no células pequeñas se avecinan cambios muy importantes. Por un lado, ya forma parte de las guías clínicas internacionales el TAC de baja intensidad como estudio de screening en pacientes con factores de riesgo. El empleo de estos estudios va a implicar un aumento muy importante en el número de pacientes con lesiones tempranas, con nódulos pulmonares sospechosos, siendo su resolución un desafío para los servicios de salud. En segundo lugar, en los últimos años 
hemos asistido a un cambio en el tratamiento de los pacientes con lesiones tempranas T1-T2 N0, pacientes que antes se trataban exclusivamente con cirugía hoy contamos con un tratamiento con resultados similares, pero con mucho menos complicaciones. Esta situación ha hecho que sea difícil poder contar con estudios randomizados, dado que en el momento en que los pacientes analizan el consentimiento informado raramente optan por el tratamiento quirúrgico, que implica una toracotomía, una hospitalización y los riesgos de una cirugía mayor, prefiriendo un tratamiento ambulatorio, que requiere de entre 1 y 5 fracciones, con una duración de una hora cada una y durante 1 a 7 días.

Esos cambios implican un desafío para los centros de radioterapia que deberían comenzar a desarrollar estas técnicas de tratamiento sofisticadas que son el presente del tratamiento de los pacientes con cáncer de pulmón no células pequeñas en etapas tempranas. ¿Estamos acercándonos a lo que llamamos el tratamiento conservador del cáncer de pulmón?

Los cambios que se avecinan son tan importantes que ya en algunas publicaciones se está estimando la reducción en el número de cirugías por cáncer de pulmón en un futuro cercano y un cambio en la fuerza de trabajo ${ }^{63}$.

\section{Bibliografía}

1.- SIEGUEL R, MILLER K, JAMAL A. Cancer Statistic, 2015. CA Cancer J Clin 2015; 65: 5-29.

2.- MINISTERIO DE SALUD. Registros Poblacionales de Cáncer de Chile, quinquenio 2003-2007, Unidad de Vigilancia de Enfermedades no Transmisibles, departamento de Epidemiología, división Planificación Sanitaria, 2012.

3.- JANSSEN-HEIJEN M. Epidemiology of Lung Cancer En: Syrigos K, Nitting C, Roussos C: Tumors of the Chest. Biology, diagnosis and Management. Springer Berlin Heidelberg 2006.

4.- SMITH R A, MANASSARAM-BAPTISTE D, BROOKS D, DOROSHENK M, FEDEWA S, SASLOW D, et al. Cancer Screening in the United States, 2015. A review of Current American cancer Society Guidelines and Current issues in cancer Screening. CA Cancer J Clin 2015; 65: 30-54.

5.- VERONESI G. Lung cancer Screening. The European Perspective. Thorac Surg Clin 2015; 25: 161-74.

6.- MARCUS M, RAJI O, FIELD J. Lung cancer screening: identifying the high risk cohort. J Thorac Dis 2015; 7 (S2): 156-62.

7.- NATIONAL COMPREHESIVE CANCER NETWORK $(\mathrm{NCCN})$. Clinical Practice Guidelines in Oncology
(NCCN Guidelines) Non-Small cell Lung Cancer. Version 7.2015, NCCN.org. www.nccn.org.

8.- MACBETH F R, ABRATT R P, CHO K H, STEPHENS R J, JEREMIC B. Lung cancer management in limited resource settings: guideline for appropriate goos care. IAEA clinical Guidelines. Radiotherapy and Oncology 2007; 82: 123-31.

9.- KORST R. Treatment of Non-small cell lung cancer: Surgery in Hansen H. Textbook of Lung Cancer. $2^{\text {nd }}$ edition, Informa UK Ltd, 2008.

10.- RAZ D J, ZELL J A, OU S H, GANDARA D R, ANTON-CULVER H, JABLONS D M. Natural History of stage I non-small-cell lung cancer. Implication of early detections Chest 2007; 132: 193-9.

11.- ROWEL N P, WILLIAMS C J. Radical radiotherapy for stage I/II non-small-cell-lung cancer in patients not sufficientely fit for or declining surgery. Cochrane Database Syst rev 2001; (2): CD002935. http://dx.doi. org/10.1002/14651858.cd002935.

12.- NESBITT J C, PUTNAM J B JR, WALSH G L, ROTH J A, MOUNTAIN C F. Survival in early-stage nonesmall cell-lung cancer. Ann Thorac Surg 1995; 60: 46672.

13.- BLOMGREN H, LAX I, NÄSLUND I, SVANSTRÖM R. Stereotactic high dose fraction radiation therapy of extracraneal tumors using an accelerator. Clinical experience of the first thirty-one patients. Acta Oncol 1995; 34: 861-70.

14.- CHEN H, LOUIE A. Stereotactic ablative radiotherapy and surgery: two gold standards for early-stage nonsmall-cell lung cancer? Ann Transl Med 2015; 3: 113-6.

15.- THE NATIONAL LUNG SCREENING TRIAL RESEARCH TEAM. Reduced Lung-cancer Mortality with Low dose computed tomographic screening. N Eng J Med 2011; 365: 395-409.

16.- WENDER R, FONTHAM E T, BARRERA E JR, COLDITZ G A, CHURCH T R, ETTINGER D S. American cancer Society Lung Cancer Screening Guideline. CA Cancer J Clin 2013; 1-10.

17.- SENAN S, PAUL M, LAGERWAARD F. Treatment of early-stage lung cancer detected by screening: surgery or stereotactic ablative radiotherapy? Lancet Oncol 2013; 14: e270-4.

18.- CHI A, LIAO Z, NGUYEN N P, XU J, STEA B, KOMAKI R. Systemic review of the pattterns of failure following stereotactic body radiation therapy in earlystage non-small cell lung cancer. Clinical implicatiosn. Radiat Oncol 2010; 94: 1-11.

19.- ONISHI H, SHIRATO H, NAGATA Y, HIRAOKA M, FUJINO M, GOMI K. Stereotactic body radiotherapy (SBRT) for operable stage I non-small cell lung cancer: can SRBT be comparable to surgery. Int J Radiat Oncol Biol Phys 2011; 81: 1352-8.

20.- KIM M S, KIM W, PARK I H, KIM H J, LEE E, JUNG $\mathrm{J} \mathrm{H}$, et al Radiobiological mechanism of stereotactic body radiation therapy an sterotactic radiation surgery. 
Radiat Oncol J 2015; 33: 265-75.

21.- BURNETTE B, WEICHSELBAUM R. The Inmunology of Ablative Radiation. Semin Radiat Oncol 2015; 25: 40-5.

22.- CHANG S, CHO CH, YUAN J. Radiobiology of Stereotactic Body Radiation Therapy/stereotactic Radiosurgery and the Lineal-Quadratic Model. Inte J Radiat Oncol Biol Phys 2013; 87: 18-9.

23.- VATNER R, COOPER B, VANPOUILLE C, DE MARÍA S, FORMANTI S. Combination of immunotherapy and radiation in cancer therapy. Frontiers in Oncology 2014; 4; article 325: 1-14.

24.- ILLIDGE T. Turning radiotherapy into an effective systemic Anti Cancer treatment in combination with Inmunotherapy. Clin Oncol (R Coll Radiol) 2015; 27: 696-9. doi: 10.1016/j.clon.2015.09.001. Epub 2015 Sep 26.

25.- LOUIE A, PALMA D, DAHELE M, RODRIGUES G B, SENSH S. Management of early-stage non-small cell lung cancer using stereotactic ablative radiotherapy: compressive, insights, and changing horizonts. Radiat Oncol 2015; 114: 138-47.

26.- CHANG J. Guidelines and Techniques for ImageGuided radiation therapy for Non-Small cell Lung cancer. In: Cox J, Chang J , Komaki R. Image-Guided Radiotherapy of Lung Cancer. Informa healthcare, New York, 2007.

27.- NCCN Clinical Practice Guidelines in Oncology (NCCN Guidelines $^{\mathrm{R}}$ ) Non-Small cell Lung Cancer. Version 7.2015, NCCN.org. www.nccn.org.

28.- YU J B, SOULOS P R, CRAMER L D, DECKER R H, KIM A W, GROSS C P. Comparative Effectiveness of Surgery and Radiosurgery for Stage I non-Small Cell Lung Cancer. Cancer 2015; 121: 2341-9.

29.- TIMMERMAN R, MCGARRY R, YIANNOUTSOS

C, PAPIEZ L, TUDOR K, DELUCA J, et al. Excessive toxicity whith treating central tumors in a phase II study of stereotactic body radiation therapy for medically inoperable early-stage lung cancer. J Clin Oncol 2006; 24: 4833-9.

30.- FAKIRIS A J, MCGARRY R C, YIANNOUTSOS C T, PAPIEZ L, WILLIAMS M, HENDERSON M A, et al. Stereotactic body radiotherapy for early stage non-small cell lung carcinoma: four year results of a prospective phase II study. Int J Radiat Oncol Biol Phys 2009; 75: 677-82.

31.- BRAL S, GEVAERT T, LINTHOUT N, VERSMESSEN H, COLLEN C, ENGELS B, et al Prospective riskadapted strategy of stereotactic body radiotherapy for early-stage non-small cell lung cancer. Results of Phase II trial. Int J Radiat Oncol Biol Phys 2011; 80: 1343-9.

32.- VAN DER VOORT VAN ZYP N C, PRÉVOST J B, HOOGEMAN M S, PRAAG J, VAN DER HOLT B, LEVENDAG P C, et al. Stereotactic radiotherapy with real-time tumor tracking for non-small cell lung cancer: clinical outcome. Radiother Oncol 2009; 91: 296-300.
33.- BROWN W T, WU X, FAYAD F, FOWLER J F, AMENDOLA B E, GARCÍA S, et al. CyberKnife Radiosurgery for stage I lung cancer: results at 36 months Clin lung cancer 2007; 8: 488-92.

34.- SENTHI S, LAGERWAARD F J, HAASBEEK C J, SLOTMAN B, SENAN S. Patterns of disease recurrence after sterotactic ablative radiotherapy for early stage non-small cell lung cancer: a restrospective analysis. Lancet Oncol 2012; 13: 802-9.

35.- ONISHI H, SHIRATO H, NAGATA Y, HIRAOKA M, FUJINO M, GOMBI Y, et al. Hypofractionated stereotactic radiotherapy for stage I non-small cell lung cáncer. Updated results of 257 patients in a Japanese multi-institutional study. J Thoac Oncol 2007; 2 (7 suppl 3): S94-100.

36.- NAGATA Y, HIRAOKA M, SHIBATA T, ONISHI H, KOKUBON M, KARASAWA K, et al. Stereotactic Body radiation therapy for T1 N0 M0 Non-small cell lung cancer. First reports for inoperable population of a Phase II trial by japan clinical Oncology group (JCOG 0403) Int J Radiat Oncol Biol Phys 2012; 84 (suppl): s46.

37.- PARK B, YEE C, MI LEE K. The effect of radiation on Immune response to cancer Int J Mol Sci 2014; 15 : 927-43.

38.- MCGARRY R C, PAPIEZ L, WILLIAMS M, WHITFORD T, TIMMERMAN R D. Sterotactic body radiation therapy of early-stage non-small cell lung cancer: Phase I study. Int J Radiat Oncol Biol Phys 2005; 64: 1010-5.

39.- TIMMERMAN R, PAPIEZ L, MCGARRY R, LIKES L, DESROSIERS C, FROST S, et al. Extracraneal stereotactic radioablation: results of phase I steady in medically inoperable stage I no-small cell lung cancer. Chest 2003; 124: 1946-55.

40.- CHANG J Y, BALTER P A, DONG L, YANG Q, LIAO

$Z$, JETER M, et al. Stereotactic body radiation therapy in centrally and superiolly located stage I or isolated recurrent non-cell lung cancer. Int J radiat Oncol Biol Phys 2008; 72: 967-71.

41.- MILANO M T, CHEN Y, KATZ A W, PHILIP A, SCHELL M C, OKUNIEFF P. Central thoracic lesions treated with hypofrationed sterotactic body radiotherapy. Radiother Oncol 2009; 91: 301-6.

42.- HAASBEEK C J, LAGERWAARD F J, SLOTMAN B J, SENAN S. Outcome of stereotactic ablative radiotherapy for centrally located early-stage lung cancer. J Thorac Oncol 2011; 6: 2036-43.

43.- ROWE B P, BOFFA D J, WILSON L D, KIM A W, DETTERBECK F C, DECKER R H. Stereotactic body radiotherapy for central lung tumors. J Thorac Oncol 2012; 7: 1394-9.

44.- ROWE B P, BOFFA D J, WILSON L D, KIM A W, DETTERBECK F C, DECKER R H. Sterotactic body radiotherapy for lung tumors at the pulmonary hilium. Strahlether Onkol 2010; 186: 274-9. 
45.- UNGER K, JU A, OERMANN E, SUY S, YU X. CyberKnife for hiliar lung tumors: report of clinical response and toxicity J Hematol Oncol 2010; 3: 39 doi: 10.1186/1756-8722-3-39.

46.- BABA F, SHIBAMOTO Y, OGINO H, MURATA R, SUGIE C, IWATA H, et al. Clinical outcomes of stereotactic body radiotherapy for stage I non-small cell lung cancer using different doses depending on tumor size. Radiat Oncol 2010; 5: 81-8.

47.- MAHMOOD S, BILAL H, FAIVRE-FINN C, SHAH $\mathrm{R}$. Is stereotactic ablative radiotherapy equivalent to sublobar resection in high-risk surgical patients with stage I non-small cell lung cancer? Interact Cardiovasc Thorac Surg 2013; 17: 845-53.

48.- PALMA D, VISSER D, LAGENWARD F J, BELDERBOS J, SLOTMAN B, SENAN S. Treatment of Stage I NSCLC in elderly patients: a populations-based matched-pair comparison of stereotactic radiotherapy versus surgery. Radiother Oncol 2011; 101: 2404.

49.- LAGERWAARD F J, VERSTEGEN N E, HAASBEEK C J, SLOTMAN B J, PAUL M A, SMIT E F, et al. Outcome of stereotactic ablative radiotherapy in patients with potencially operative stage I non-small cell lung cancer. Int J Radiat Oncol Biol Phys 2012; 83: 384-53.

50.- ZHENG X, SCHIPPER M, KIDWELL K, LIN J, REDDY R, REN Y, et al. Survival putcome after stereotactic body radiation therapy and surgery for stage I non-small cell lung cancer: a meta-anatysis. Int J Radiat Oncol Biol Phys 2014; 90: 603-11.

51.- CLINICAL/TRIALS.GOV (Internet). Bethesda (MD): National Library of Medicine (US) 2015 jun 2-Identifier NCT00687986. Trial of Either surgery or Stereotactic Radiotherapy for early Stage (IA) Lung Cancer (ROSEL): 28.05. 2008 Disponible en: https://clinicaltrials. gov/ct2/show/NCT00687986.

52.- CLINICAL/TRIALS.GOV (Internet). Bethesda (MD): National Library of Medicine (US), 2013 Apr 5- Identifier NCT00840749. Randomized Study to compare CyberKife to Surgical resection in stage I Non-small cell lung cancer (STARS) 2209 feb 7 (Edited 2015 apr 13) Disponible en: https://clinicaltrials.gov/ct2/show/ NCT00940749.

53.- ADVANCED TECHNOLOGY QA CENTER (Internet) St. Louis (MO): Washington Univerity in ST Louis School of medicine. 2011 May 2-ASCOG PROTOCOL Z4099: RTOG PROTOL 1021 randomized phase III study of sublobar resection (+/- brachytherapy) versus stereotactic body radiation therapy in high risk patients with stage I non-small cell lung cancer (NSCLC); 2011 May 2 (cited 2015 Apr 13): Disponible en: http.//atc. wusstl.edu/protocols/rtog/1021/1021.html.

54.- CHANG J Y, SENAN S, PAUL M A, MEHRAN R J, LOUIE A V, BALTER P, et al Stereotactic ablative radiotherapy versus lobectomy for operable stage I non-small-cell lung cancer: a pooled analysis of two randomized trials. Lancet Oncology 2015; 16: 630-7.
55.- NIEDER C, ANDRATSCHE N, GUCKENBERGER M. A pooled analysis of ablative radiotherapy versus lobectomy for operable stage I non-small cell lung cancer: is failure to recruit patients into randomized trials also an answer to the research question? Ann Thor Med 2015, 3: 148.

56.- RUSTHOVEN C, KAVANAGH B D, KARAM S. Improved survival with sterotactic ablative radiotherapy (SRABT) over lobectomy for early stage non-small cell lung cancer (NSCLC): addressing the fallout of disruptive randomized data. An Transl Med 2015; 3 : 149-55.

57.- RADIATION THERAPY ONCOLOGY GROUP [Internet]. Philadelphia: RTOG. 2015 Jun 8-RTOG 0813 Protocol Information, Seamless Phase I/II Study of Stereotactic Lung Radiotherapy (SBRT) for Early Stage, Centrally Located, Non-Small Cell Lung Cancer (NSCLC) in Medically Inoperable Patients; 2013 Sep 5 [cited 2015 Apr 13]; Disponible en: https://www. rtog.org/ClinicalTrials/ ProtocolTable/StudyDetails. aspx? study $=0813$.

58.- RADIATION THERAPY ONCOLOGY GROUP [Internet]. Philadelphia: RTOG. 2014 Mar 6 - RTOG 0915 Protocol Information, A Randomized Phase II Study Comparing 2 Stereotactic. Disponible en: https://www. rtog.org/.../ProtocolTable/StudyDetails.aspx?action.

59.- GOLD M K, DONINGTON J, LYNCH W R, MAZZONE P, MIDTHUUN D, NAIDICH D, et al. Evaluation of individuals with pulmonary nodules whwn is it lung cancer? Diagnosis and management of lung cancer. $3^{\text {ed }}$ ed. American College of Chest Physician evidence based clinical practice guideline. Chest 2013; 143 (5 Suppl): e935-e1205.

60.- HIRAKI Y, MIMURA H, GOBARA H, IGUCHI T, FUJIMURA H, SAKURAI J, et al. CT fluoroscopy guided biopsy of 1,000 pulmonary lesions performed with 20-gauge coaxial cutting needle diagnostic yield and risk factors for diagnostic failure. Chest 2009; 136 : 1612-7.

61.- GUCKENBERGER M, ALLGÄUER M, APPOLD S, DIECKMANN K, ERNST I, GANSWINDT U, et al. Safety an efficacy of sterotactic body radiotherapy for stage I non-small cell lung cancer in routine clinical practice: a patterns of care and outcome analysis. J Thorac Oncol 2013; 8: 1050-8.

62.- LOUIE A V, SENAN S, PATEL P, FERKET B S, LAGERWAARD F J, RODRIGUES G B, et al. When is Biopsy-Proven Diagnosis necessary before Sterotactic Ablative radiotherapy for lung cancer? Chest 2014; 146 : 1021-8.

63.- EDWARDS J P, DATA I, HUNT J D. Forecasting the impact of sterotactic ablative radiotherapy for earlystage lung cancer on the thoracic surgery workforcw. Eur J Cardiothorac Surg 2016, jan 21 pii (Epub ahead of print).

64.- VALLE L, JAGSI R, BOBIAK S, ZORNOSA C, 
D'AMICO T, PISTERS K, et al. Variation in definitive Therapy for localized Non-small cell lung cancer among national comprehensive cancer network Institutions. Int J Radiat Oncol Biol Phys 2016; 94: 391-7.

65.- SHARABI A B, TRAN P T, LIM M, DRAKE C G, DEWEESE T L. Stereotactic radiation therapy combined with immunotherapy: augmenting the role of radia- tion in local and systemic treatment. Cancernetwork, home of the journal oncology. http://cancernetwork.com 2016.

66.- NUYTTENS J. Sterotactic radiotherapy for lung tumors. In: Gaya A and Mahadevan A Eds Sterotactic Body Radiotherapy, a practical Guide. Springer-Verlag London 2015.

Correspondencia a:

Dr. Alejandro Santini B.

Centro Oncológico Antofagasta, Chile.

Email: alejandro.santini@gmail.com 